\title{
The Acoustic Environment of the NASA Glenn 9- by 15-Foot Low-Speed Wind Tunnel
}

\author{
David B. Stephens* \\ NASA Glenn Research Center, Cleveland, Ohio, 44135.
}

\begin{abstract}
The 9- by 15-Foot Low Speed Wind Tunnel is an acoustic testing facility with a long history of aircraft propulsion noise research. Due to interest in renovating the facility to support future testing of advanced quiet engine designs, a study was conducted to document the background noise level in the facility and investigate the sources of contaminating noise. The anechoic quality of the facility was also investigated using an interrupted noise method. The present report discusses these aspects of the noise environment in this facility.
\end{abstract}

\section{Introduction}

$\mathrm{T}$ HE 8- by 6-Foot Supersonic Wind Tunnel (8x6 SWT) at NASA Glenn Research Center (GRC) was completed in 1949 as an open loop propulsion tunnel for testing ramjets and other high-speed air breathing engines. The operation of the tunnel and test engines was found to produce objectionable noise for miles around the facility, so an immediate and comprehensive acoustic mitigation upgrade was conducted during the first half of $1950 .{ }^{1}$ This upgrade included the addition of a series of Helmholtz resonators tuned to reduce frequencies as low as $5 \mathrm{~Hz}$, building a large muffler downstream of the 8x6 SWT diffuser and the addition of acoustic panels in various parts of the facility. The 8x6 SWT was converted into a closed loop tunnel with the addition of a return leg in 1956. The 9- by 15-Foot Low Speed Wind Tunnel (9x15 LSWT) was built in the return leg of the 8x6 SWT in 1969. ${ }^{2}$ The 9x15 LSWT was designed for performance testing of V/STOL aircraft models, but with the addition of the current acoustic treatment in 1986, the tunnel has been used principally for acoustic and performance testing of aircraft propulsions systems. A number of reports overview the facility including aerodynamic test capabilities and acoustic quality. ${ }^{3,4,5,6,7,8}$ A sketch of the $8 \times 6 / 9 \times 15$ wind tunnel complex is given as Figure 1 .

Testing in the 9x15 LSWT has included model turbofans and propellers for both NASA research projects and external customers. ${ }^{9,10,11}$ Current turbofan models are powered by the NASA Ultra-High Bypass drive rig. ${ }^{12}$ Recent and future turbofan models being evaluated are advanced designs incorporating technologies such as reduced fan tip speeds, increased bypass ratios and advanced acoustic liners. Model engines utilizing these technologies in combination are significantly quieter than earlier models. In the past, most turbofan models have been tested at a tunnel speed of Mach 0.1 in order to limit the tunnel background noise level. This speed was determined to be fast enough to provide the desired aerodynamically clean inflow condition at the fan face, for models where the nacelle inlet was sufficiently long. Future turbofan engines are expected feature shorter inlets to reduce weight and drag such that less flow conditioning from the nacelle is available. To achieve the desired inflow at the fan face, the tunnel must be operated at a higher freestream velocity that more closely matches the intended flight speed. This raises the background noise level in the facility, making acoustic measurements of advanced turbofans increasingly challenging.

The present report describes the current background noise levels in the 9x15 LSWT and some of the known noise sources. It briefly describes a series of measurements made on the noise due to airflow over perforated sheet metal, such as that found in the 9x15 LSWT test section. A series of reverberation time measurements made using an interrupted noise method is also presented. These tests combine to serve as a current baseline for the noise environment in the 9x15 LSWT and outline some points of emphasis for improving the facility.

\footnotetext{
*Research Aerospace Engineer, Acoustics Branch, MS 54-3, 21000 Brookpark Road. AIAA Lifetime Member.
} 


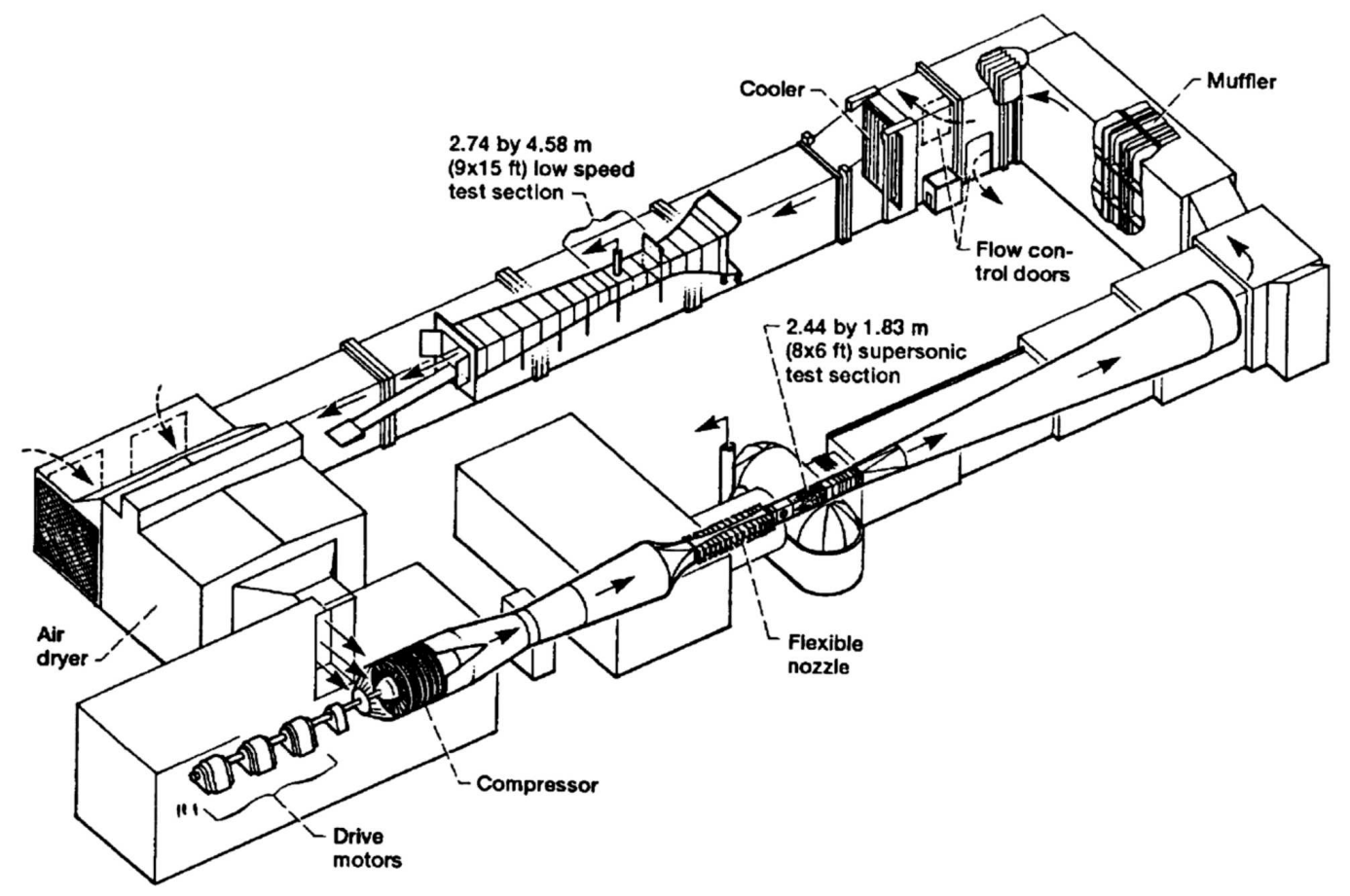

Figure 1. Schematic of the $8 \times 6 / 9 \times 15$ wind tunnel complex.

\section{9x15 LSWT Noise Levels}

An extensive set of background noise measurements was acquired in February and March 2012 while the tunnel was empty (except for microphone probes and the requisite pressure and temperature rakes). This data set was acquired to support an acoustic assessment that was performed by Jacobs Engineering Group, based in Tullahoma, Tennessee. It also served to document the current background noise levels. The 9x15 LSWT test section as it was tested is shown in Figure 2. A traversing track and microphone holder with three sensors is visible at the right side of the photograph. This instrumentation serves as the main acoustic measurement system for the facility.

\section{II.A. Background Noise in the Test Section}

As part of normal acoustic testing procedures, background noise levels in the test section are periodically recorded. ${ }^{8}$ Measurements taken many years apart have been found to be very similar, suggesting that the major sources of background noise in the facility are not affected by normal tunnel operations. Acoustic measurements from the center microphone on the traverse at the midpoint of the test section are plotted in Figure 3. All spectra in this report are presented as narrowband pressure spectral densities (PSD) in decibels referenced to 20 micro-Pascals. The background noise was found to be largely uniform throughout the wind tunnel test section, varying only marginally from front to back or across the test section.

\section{II.B. Increase in Tunnel Noise Level with Mach Number}

In order to better understand the noise environment in the tunnel, the scaling of the noise with free stream Mach number was investigated. Without knowing precisely the noise source mechanisms that generate the inflow noise measured when the tunnel is empty, we consider that aerodynamically generated sound scales with Mach number as,

$$
\overline{p^{2}} \propto M^{n},
$$

or

$$
10 \log _{10}\left(\overline{p^{2}}\right) \propto n 10 \log _{10}(M),
$$




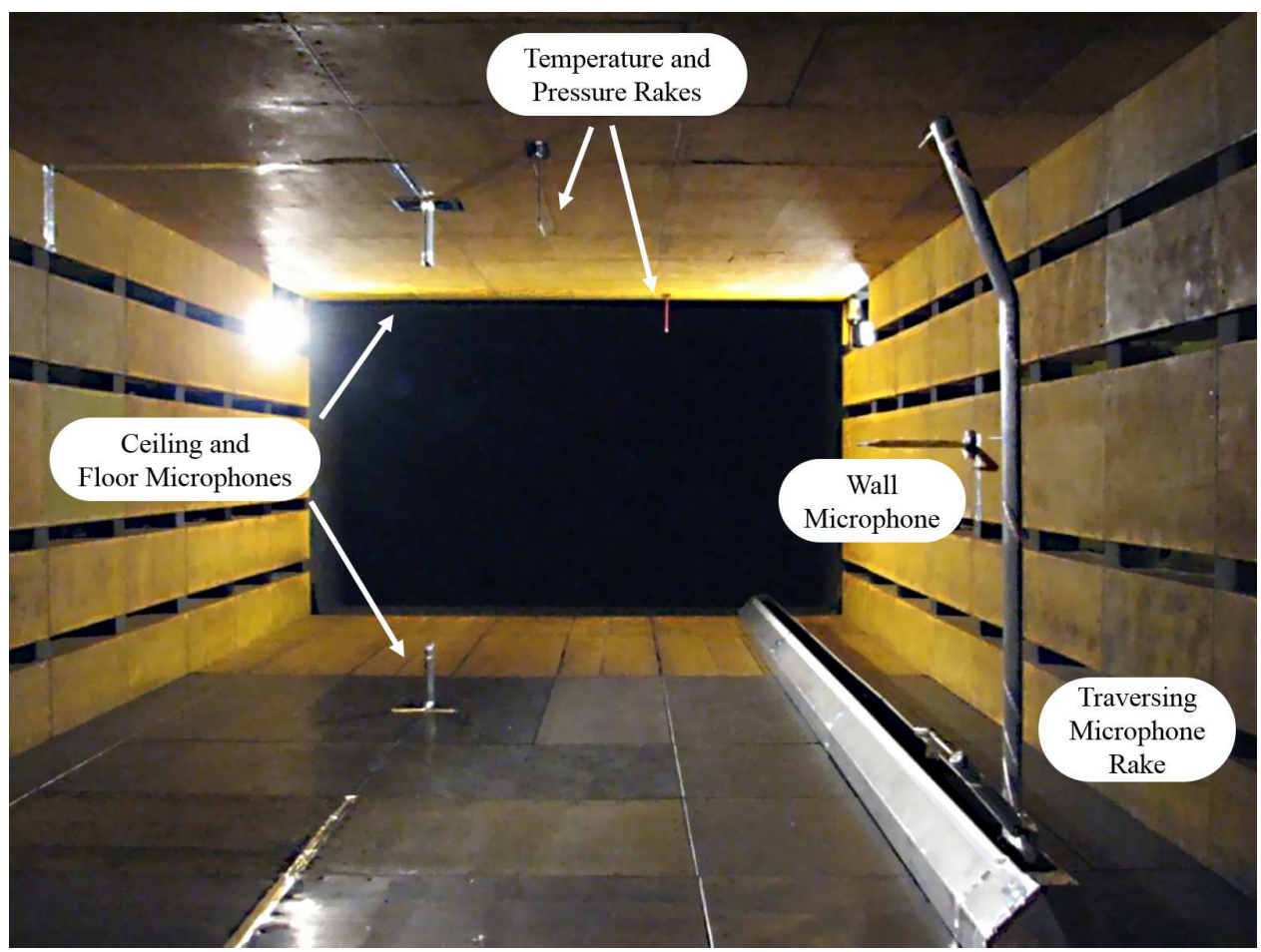

Figure 2. Empty $9 \times 15$ LSWT in configuration used for background noise measurement. View is from the downstream end of the test section, looking upstream.

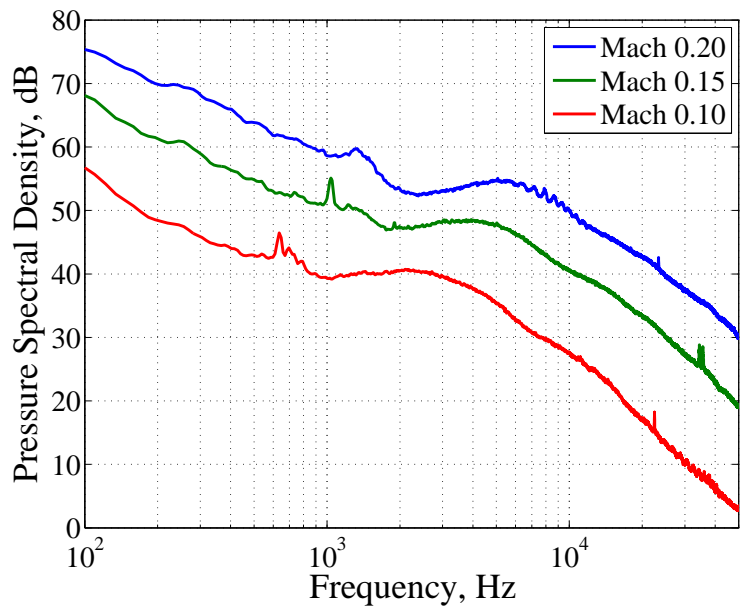

Figure 3. Background noise level measured in the 9x15 LSWT.

where $\overline{p^{2}}$ is the variance of the acoustic pressure, $M$ is the tunnel Mach number and $n$ is an exponent that we wish to determine. Such an analysis was performed on a one-third octave band basis by Duell et al., ${ }^{13}$ and values of $n$ between 5 and 7 were found, depending on frequency.

Regarding the present data set, as seen in Figure 3, the narrow-band spectra scale in both amplitude and frequency as Mach number increases. It was therefore of interest to consider the noise scaling as a function of frequency normalized by Mach number $f^{\prime}=\frac{f}{M}$. If this were a situation with an obvious length scale, a Strouhal number would be appropriate to use, but the wind tunnel has many different length scales and none of them were changed for this data set.

Plots of the normalized spectra are given in Figure 4 (a). The spectral features of the measured noise are seen to be largely aligned with this normalization, although some irregularities are apparent. Based on their prior experience with large scale acoustic wind tunnels, ${ }^{13}$ the consultants from Jacobs Engineering Group 
advised NASA that the major contributor to the noise spectra at Mach 0.20 above $2 \mathrm{kHz}$ (as seen in Figure 3 ) was likely due to airflow over the perforated plate of the tunnel walls. This corresponds to the portion of the normalized sound spectra with $f^{\prime}>13,000$, as seen in Figure 4 (a).

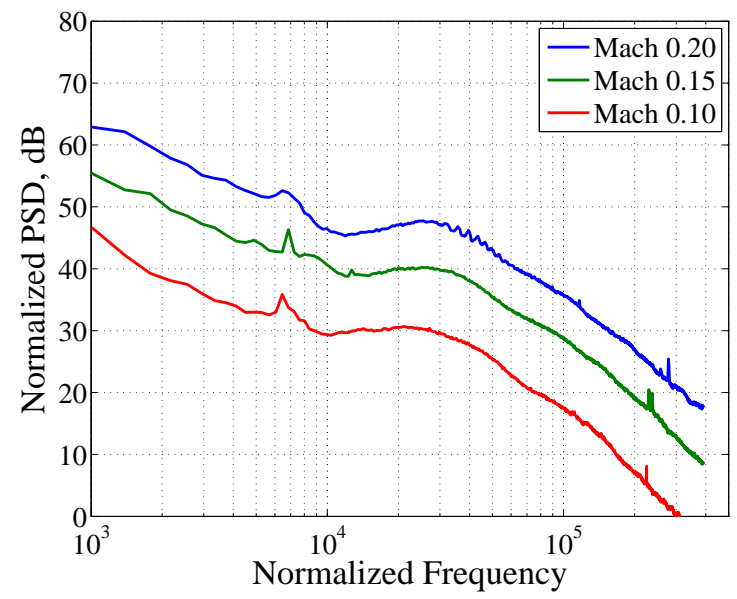

(a)

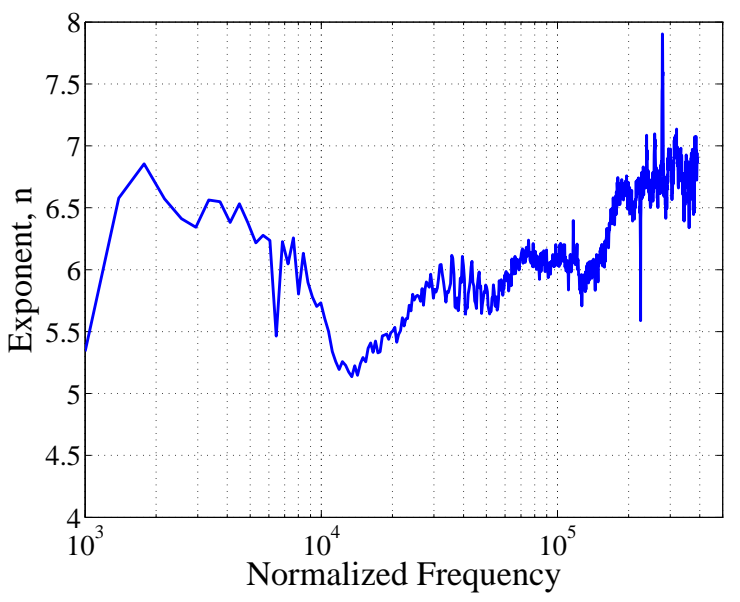

(b)

Figure 4. Effect of tunnel Mach number on background noise. (a) Normalized Spectra. (b) Mach Number Exponent.

The spectral amplitude shown in Figure 4 (a) has been scaled by Mach number in order to preserve energy. That is, changing variables from $f$ to $f^{\prime}$ is expressed as

$$
\overline{p^{2}}=\int \Phi(f) d f=\int M \Phi\left(f^{\prime}\right) d f^{\prime},
$$

since $M d f^{\prime}=d f$, and $\Phi$ represents the pressure spectral density, with units of Pasals squared per Hertz. The change of variable is substituted into Equation 2 to get,

$$
10 \log _{10}\left(M \Phi\left(f^{\prime}\right)\right) \propto n\left(f^{\prime}\right) 10 \log _{10}(M)
$$

or

$$
10 \log _{10}\left(\Phi\left(f^{\prime}\right)\right)+10 \log _{10}(M) \propto n\left(f^{\prime}\right) 10 \log _{10}(M) .
$$

In order to find $n\left(f^{\prime}\right)$, a least squares method was used to fit a line to the three data points available (that is, the left side of Equation 5) at each normalized frequency $f^{\prime}$. The slope of this line is $n\left(f^{\prime}\right)$. The result is shown in Figure 4 (b). Scaling is seen to range between about $n=5$ and $n=7$, which is consistent with the findings of Duell et al. ${ }^{13}$ A minimum is evident at about $f^{\prime}=13,000$, but this seems to be due to the imperfect alignment of the particular local maxima in the spectral density. The scaling with Mach number aligns most, but not all of the features in the spectra. The peaks in the spectral density (seen in Figure 4 (a)) occur at roughly $f^{\prime}=26,000$, and the scaling exponent $n$ is roughly 5.8 at this normalized frequency.

A second method for estimating the velocity scaling exponent for the roughness portion of noise was found by fitting a third order polynomial to the pressure spectral density at each velocity, as shown in Figure 5 (a). Assuming that each of the polynomials is a good representation of the roughness noise spectra, they can each be integrated to get overall sound pressure level (OASPL). This result is given in Figure 5 (b), where the three values of OASPL are seen to be quite linear against Mach number, with a slope of $n=5.6$. The expected value of scaling for dipole sources is $6,{ }^{14}$ so 5.6 and 5.8 could be considered fairly reasonable. This value helps support the idea that roughness noise is a major contributor to the inflow noise level in the test section. Deviations from $n=6$ scaling may be due to Reynolds number effects (such as changes in the boundary layer profile from the front to rear of the test section), air leaking into the test section, or other complications. 


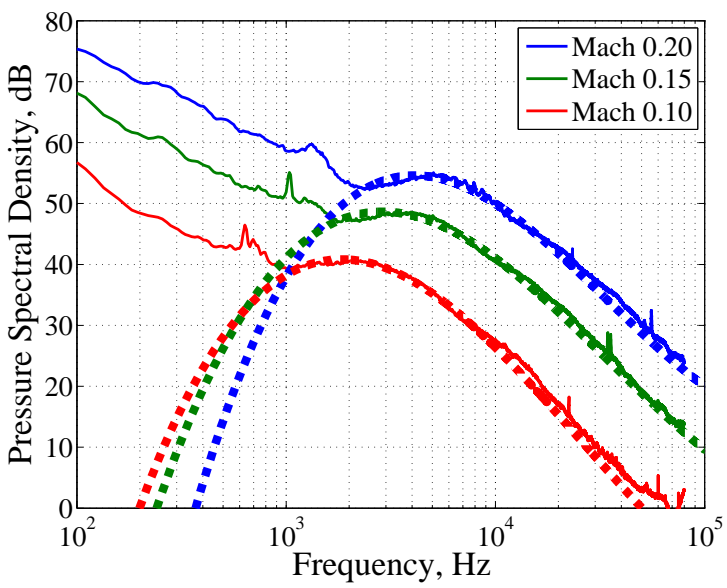

(a)

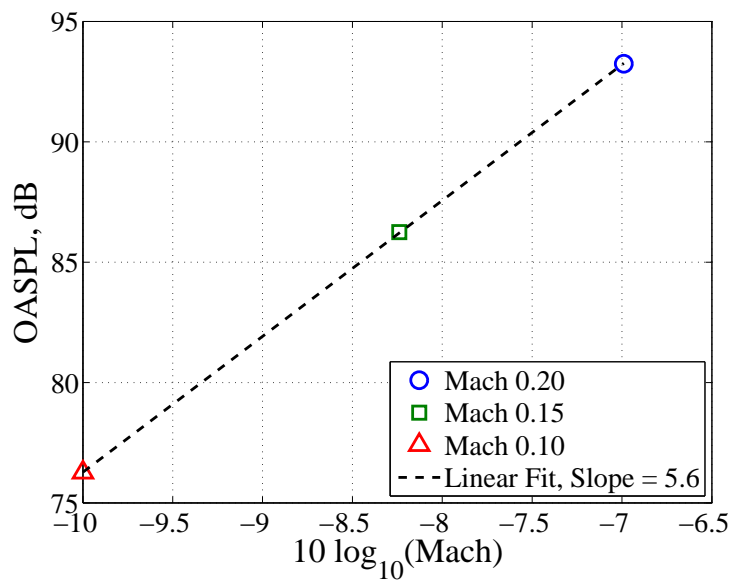

(b)

Figure 5. Roughness noise curve fits to measured 9x15 LSWT background noise. (a) Thirdorder polynomials overlain on pressure spectral density measurements. pressure level calculated from polynomials vs test section Mach number.

(b) Overall sound

\section{II.C. Noise Measurements from the Tunnel Loop}

Prior to early 2012, acoustic measurements in other parts of the wind tunnel (outside of the test section) had not been made in many years. A thorough investigation of the noise levels throughout the wind tunnel loop was therefore conducted as part of the background noise assessment. With the test section empty of any experiment, the tunnel was run at a number of airflow speeds using a variety of different flow control mechanisms. A set of microphones was used to measure the noise in key locations. A subset of the collected data is presented in this report, and a schematic showing the locations of these microphone in the tunnel loop is given in Figure 6.

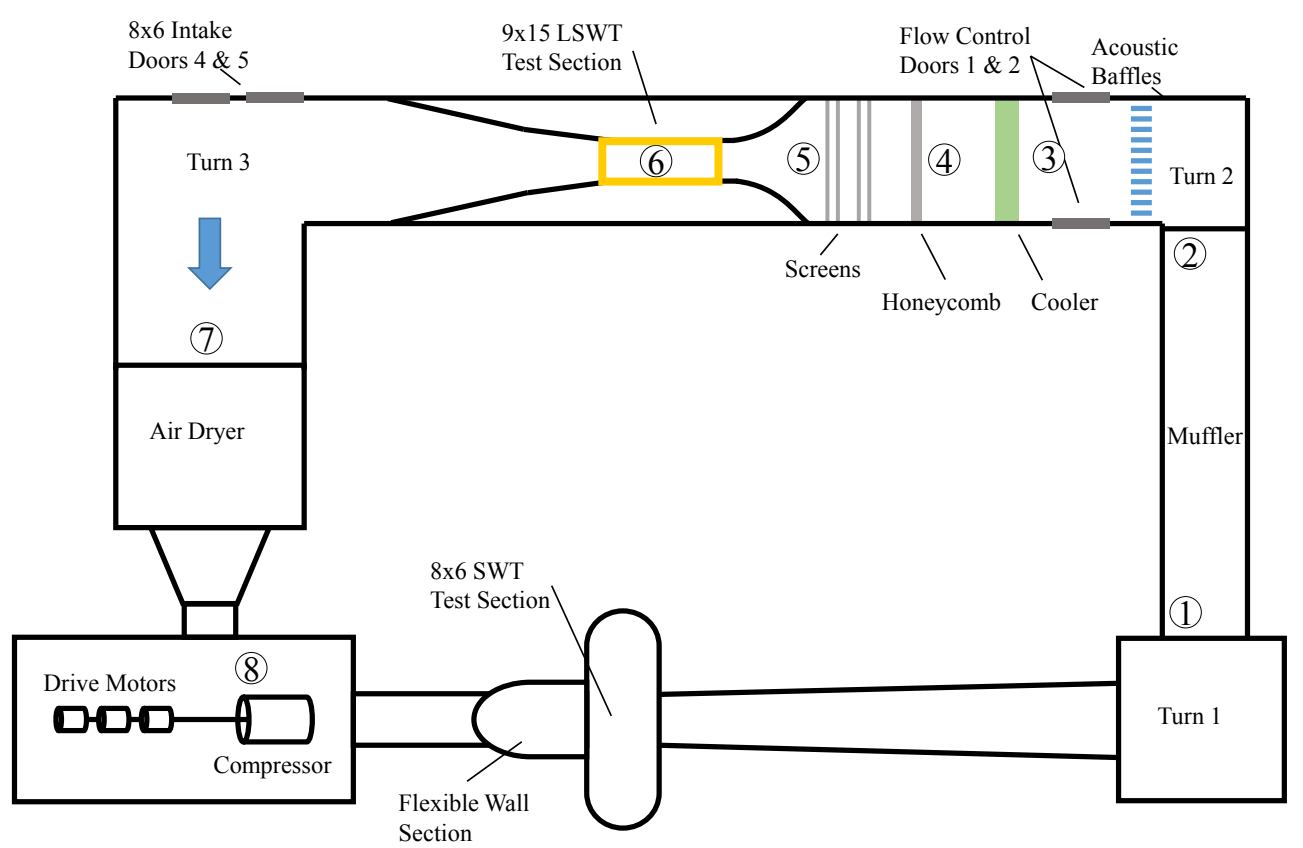

Figure 6. Some of the microphone locations used in the background noise assessment of the 8x6 SWT / 9x15 LSWT Circuit. Not to scale. 
Starting from the upstream portion of the 9x15 LSWT, Figure 7 (a) shows the noise drop across the muffler, from microphone locations 1 and 2 in Figure 6. The noise at the muffler exit is roughly 20 to 25 $\mathrm{dB}$ lower at the outlet than the inlet at all frequencies. When the muffler was first built, a pair of $38 \mathrm{~cm}$ (15 inch) speakers was used to measure the performance of the bulk absorber portion of the muffler. ${ }^{1}$ It was documented to have about $40 \mathrm{~dB}$ of attenuation across a wide range of frequencies (see Figure 40 in referenced report). The smaller level measured now may be due to self-noise in the facility limiting the noise floor of the present measurement, or due to degradation of the fiberglass lining. Repeating the speaker testing without airflow would serve to assess the quality of the acoustic treatment, but it is clear that the muffler is still quite effective at reducing noise in the tunnel loop.

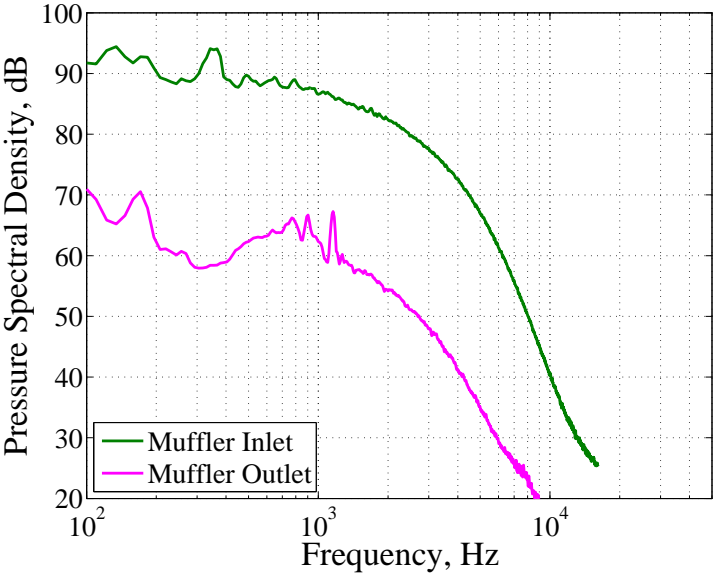

(a) .

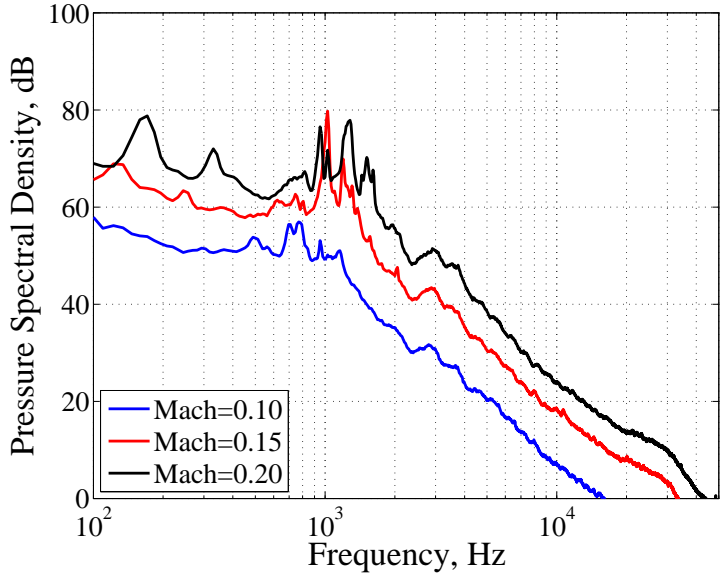

(b)

Figure 7. Noise measurements from the 9x15 loop. (a) Noise measured across the tunnel muffler. The microphone locations were those numbered 1 and 2 in Figure 6. (b) Noise due to Doors 1 \& 2, measured upstream of cooler in 9x15 LSWT at microphone location 3 from Figure 6.

The muffler exhausts into Turn 2 dumps into a $90^{\circ}$ turn without any guide vanes. The air flow then passes Doors $1 \& 2$, as indicated in Figure 6 . These doors move horizontally along a track and are remotely actuated to fine-tune the air velocity in the tunnel loop. They were built for 9x15 tunnel operations, replacing vertical doors used for $8 \mathrm{x} 6 \mathrm{SWT}$ operations. Their designed function is as exhaust doors and the total pressure in this location is above atmospheric. Fine-tune control of the tunnel air speed was improved with modifications to the electric drive system and procedural changes in the mid $1990{ }^{\prime} \mathrm{s}^{15}{ }^{16}$ Figure 7 (b) shows the noise measured upstream of the cooler, near Doors 1 \& 2. Compared with Figure 7 (a), several additional tones were observed. At Mach 0.20, low frequency tones at $170 \mathrm{~Hz}$ and $340 \mathrm{~Hz}$ are observed. Substantial unwanted noise is also generated between $900 \mathrm{~Hz}$ and $1200 \mathrm{~Hz}$, probably due to leakage out of the doors. Changing the door position between closed and $14 \%$ open (while holding the test section Mach number constant by changing the compressor speed) modifies the noise somewhat but does not reduce it. The noise may be due to airflow over the door machinery and structure, or it may be due to air leaking out of the tunnel around the flow control doors. This noise from Doors $1 \& 2$ is measured in the test section as a local maxima in all three spectra of Figure 4 at $f^{\prime}=6400$.

Proceeding downstream in the loop, the noise upstream of the test section is given as Figure 8 (a). There is some reduction in the noise from upstream as the sound passes through the cooler, but there is no acoustic treatment between the cooler and flow conditioning section. The upstream noise around $1500 \mathrm{~Hz}$ is smoothed out as it convects through the contraction, as measured by the wall mounted microphone in the test section.

Recordings were made from microphones in the 9x15 LSWT diffuser, but all showed high unsteady pressure levels which were believed to be hydrodynamic. The microphone stands were frequently observed via video camera to be buffeted by unsteady airflow, which is consistent with the separated flow expected from a diffuser with an excessively wide diffusion angle, as the present diffuser is known to have.

The sound pressure spectra measured far downstream of the test section is shown in Figure 8 (b), along with a noise measurement from the compressor plenum. The diffuser empties back into the 8x6 SWT return 


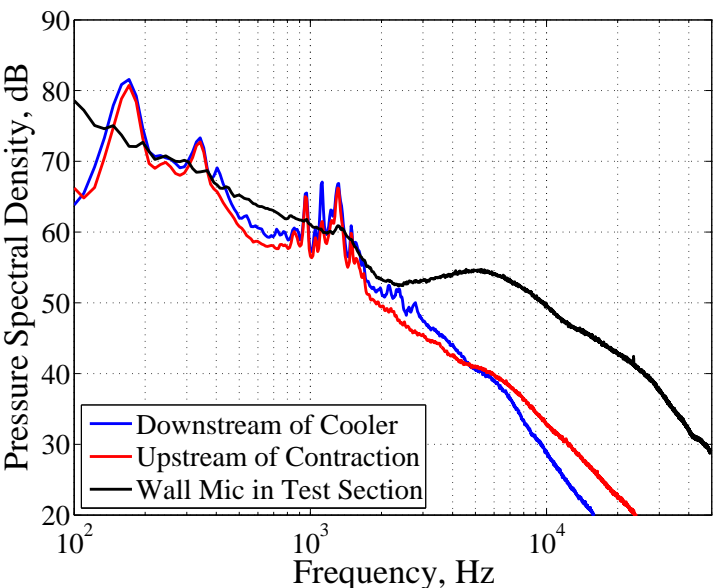

(a)

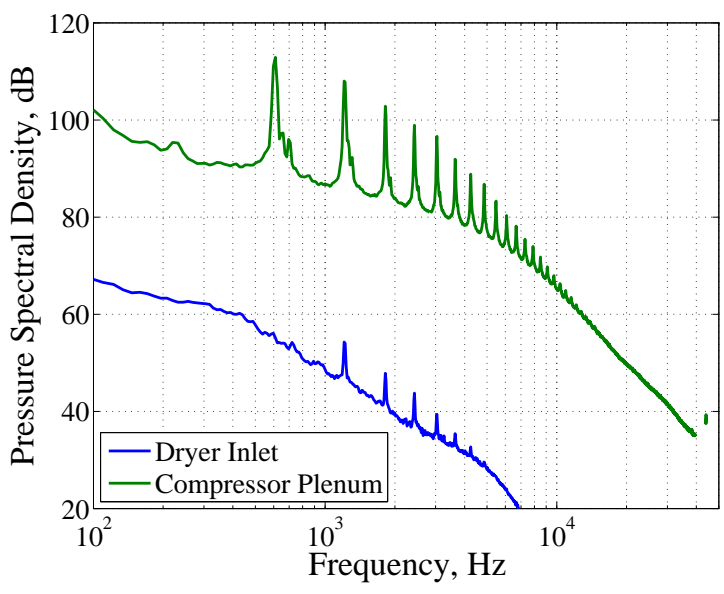

(b)

Figure 8. Noise measurements from the 9x15 loop. (a) Noise measurements from upstream of the test section, from microphone locations 4,5 and 6 in Figure 6. (b) Sound measured upstream of the air dryer and near the compressor, at locations 7 and 8 shown in Figure 6.

leg, then the flow makes a turn to enter the air dryer. As expected, the loudest sound level is recorded in the compressor inlet plenum. This area has 2 inch deep acoustic treatment that was installed in the 1950 noise mitigation effort. The 1940's era 7-stage compressor produces a very nice family of tones, but the important point is that the air dryer effectively attenuates the sound propagating upstream. A few tones can be seen in the sound spectrum recorded at the dryer inlet, however they are not observed in the test section. There is very little acoustic treatment between the end of the 9x15 LSWT test section and the air dryer inlet, and the velocity at the air dryer inlet is very small, owing to the large cross-sectional area of the building at that location. Due to the combined insertion loss of the muffler and the air dryer, no drive noise is measured in the 9x15 LSWT test section.

\section{Roughness Noise Testing}

Using the data described in the previous section, Jacobs Engineering Group Inc. conducted an assessment of the noise levels in the 9x15 LSWT. This study concluded that a major contributor to the noise in the test section of the tunnel is due to the tunnel air flow over the rough walls of the test section. These walls are formed by dozens of individual boxes built of perforated steel plate over a $33 \mathrm{~cm}$ (13") deep bulk Kevlar sound absorbing material. This steel plate is $0.16 \mathrm{~cm}$ thick $(16 \mathrm{ga})$ and perforated with 0.32 -cm-diameter $\left(1 / 8^{\prime \prime}\right)$ holes creating an open area of 40 percent, backed by a 20 -mesh screen. There are four slots in each of the two sidewalls and many seams and gaps between acoustic boxes that contribute to the rough surface.

In order to reduce the roughness noise encountered in the test section, a hydrodynamically smooth but acoustically transparent material was sought. This material would cover the perforated plate on the flow side of the test section. A facility for measuring the noise due to a rough surface was also sought, and a contract with Virginia Tech was established to utilize the wall-jet roughness noise facility at the university. ${ }^{17}$ A set of $30 \mathrm{~cm}$ by $60 \mathrm{~cm}(1 \mathrm{ft}$ by $2 \mathrm{ft}$ ) perforated plate samples was built and tested in 2013. A report on this roughness noise test was presented by Alexander and Devenport. ${ }^{18}$ An improved test was conducted in 2014, which added a $33 \mathrm{~cm}$ (13 inch) deep plywood box below the sample being tested. This box contained the same Kevlar bulk absorber used in the 9x15 LSWT. The results presented here are from the 2014 experiment.

The noise due to flow over a sample of the perforate used in the 9x15 LSWT is plotted in Figure 9. The spectral shape is seen to be largely similar to that measured in the $9 \mathrm{x} 15$, with a large local maxima at middle frequencies. The maximum to minimum velocity ratio is smaller for this data set, only 1.5 compared with 2 for the tunnel data. Also, the tunnel measurements may be smoother because the boundary layer changes significantly from the front to the back of the test section, leading to a much wider range of turbulence length and velocity scales that interact with the perforate, compared with the roughness noise experiment where the sample is specifically sized and placed to experience uniform flow. 


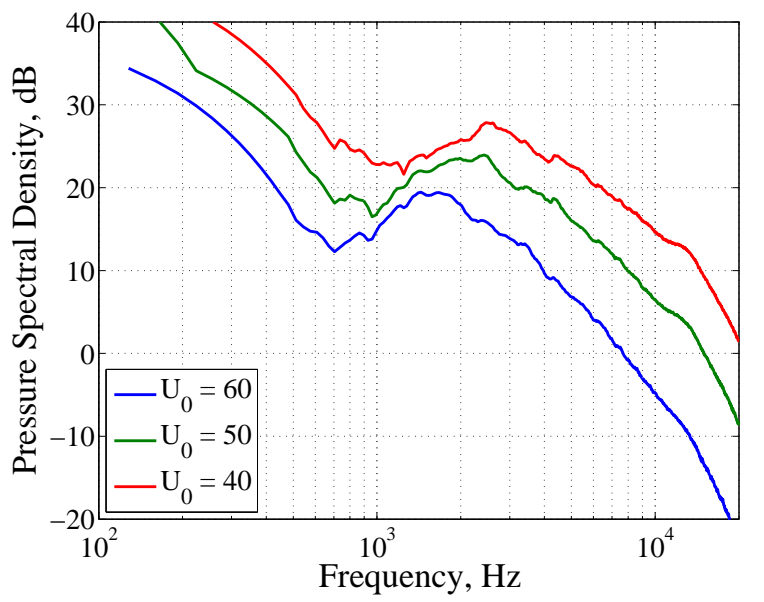

Figure 9. Noise due to flow over a perforated plate mounted over a bulk acoustic absorber.

The two methods for finding Mach number scaling discussed in Section II.B were repeated with the roughness noise test data from Virginia Tech. First, the spectra are plotted against normalized frequency $f^{\prime}$, shown in Figure 10 (a). This aligns the majority of the spectral features, but noticeably misaligns the second hump seen at $15 \mathrm{kHz}$ in Figure 9. It is not currently known what the cause of this spectral feature is, but it seems as though it does not change frequency with increasing flow speed. Using Equation 5, the frequencydependent exponent $n\left(f^{\prime}\right)$ was found and is plotted in Figure 10 (b). At the peak noise normalized frequency of $f^{\prime}=40,000$, the exponent is seen to be approximately 6 , although with considerably more irregularity than was found in the wind tunnel data.

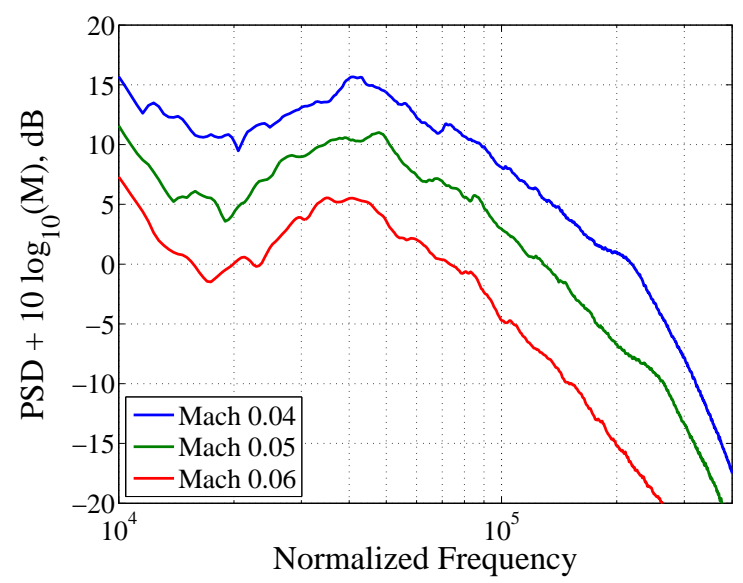

(a)

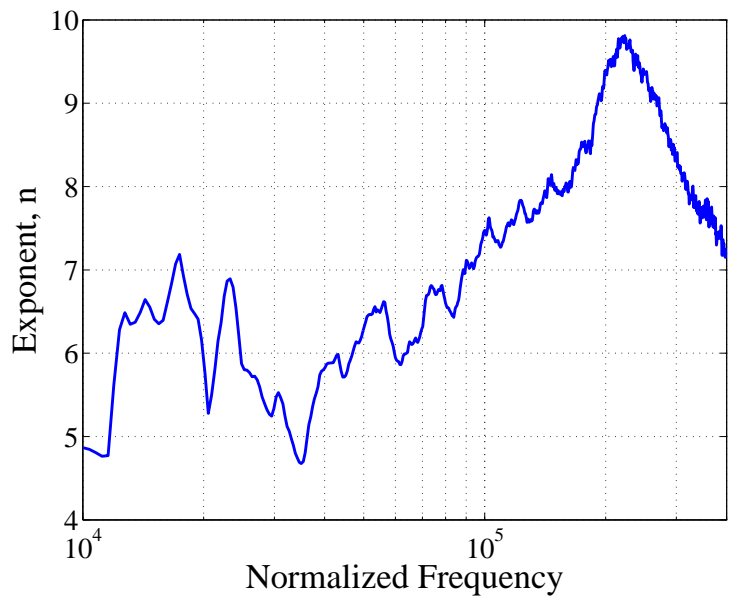

(b)

Figure 10. Finding frequency-dependent Mach number scaling exponent for roughness noise experiment. (a) Noise due to flow over a perforate plate sample, plotted against normalized frequency. (b) Frequency dependent Mach number scaling measured for perforated plate noise.

The curve-fit method was exercised again with parabolas fit to the main peak of the spectra, see Figure 11 (a). This is somewhat crude, and many models of roughness noise have been proposed, as summarized by Liu and Dowling. ${ }^{19}$ However these models typically require detailed information about the near wall flow, which has not yet been investigated for flow over the perforated plate with acoustic bulk absorber. Also, most models are based on discrete roughness elements on top of a solid surface, and still may not give satisfactory agreement with measured data without adjusting parameters. The area under each parabola can be integrated to give OASPL, and these values are plotted in Figure 11 (b). A linear fit to these points gives a value very close to 6 , confirming dipole sound as the dominant source mechanism at these frequencies. The higher and lower frequency sound source mechanisms are not known at this time. The roughness noise test 
program is being used to evaluate other surface treatments that may have reduced roughness noise compared to the bare perforate.

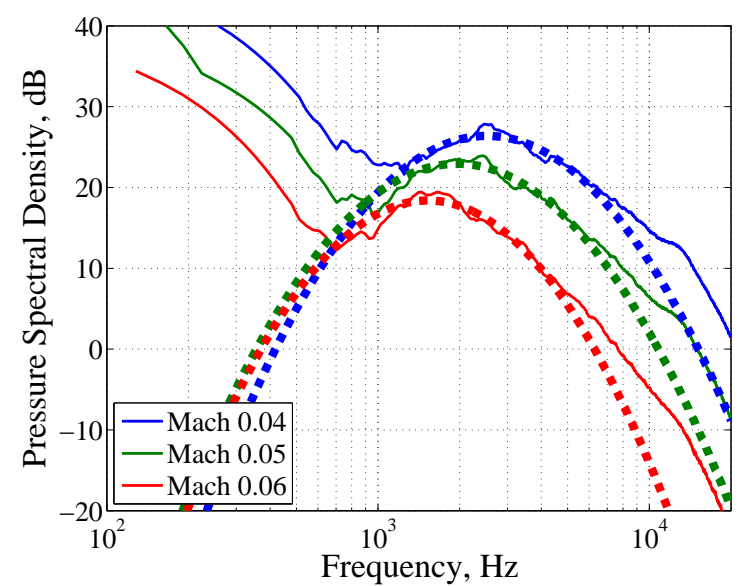

(a)

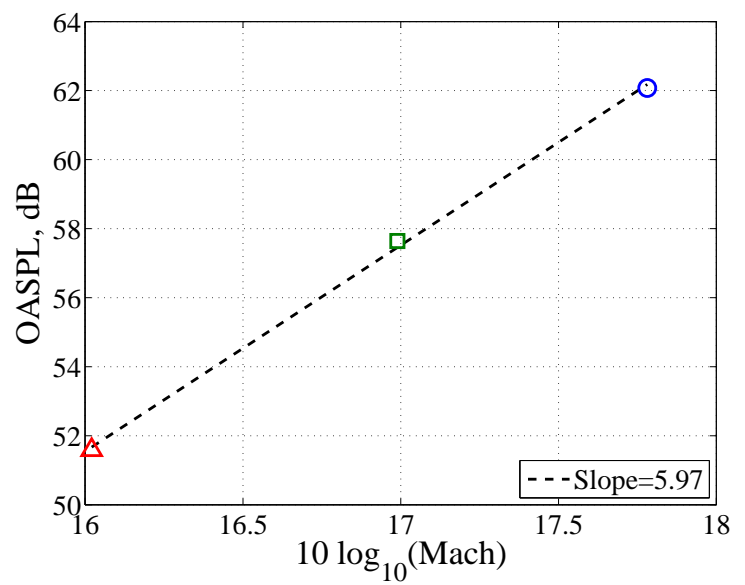

(b)

Figure 11. Reverberation time measurements at different frequencies. (a) Curve fit to perforate plate roughness noise. (b) Overall sound pressure level calculated from polynomials vs Mach number above sample.

\section{Reverberation Time Measurements}

In addition to the background noise of the facility, it was important to document the anecohic quality of the test section. The most thorough investigation of the anechoic quality of the acoustic treatment in the 9x15 LSWT was completed in 1988 and documented by Dahl and Woodward. ${ }^{6}$ Their evaluation consisted of a series of measurements, including a draw-away test to check spherical spreading and a time-delay spectrometry test to educe the impedance of the acoustic liner. The results showed the acoustic treatment had an absorption coefficient of more than 0.95 at frequencies between $250 \mathrm{~Hz}$ and $4 \mathrm{kHz}$. This value indicates that reflections would be expected to be at least $13 \mathrm{~dB}$ below the incidence noise level.

Measurements of the reverberation time in the 9x15 LSWT were made using a CESVA FP121 dodecagon speaker, largely following the specifications for interrupted noise testing described in ISO $354 .{ }^{20}$ The speaker was kept at the typical model mounting location while the traversing microphone was located at a variety of positions from the downstream edge of the test section to the upstream end. The acoustic time histories were filtered on a one-third octave band basis. A typical result is given in Figure 12 (a), which shows a varied and complicated response for four different one-third octave band center frequencies. At $630 \mathrm{~Hz}$ both early and late reflections are observed. The early reflections quickly decay to about $12 \mathrm{~dB}$ below the starting sound level whilethe sound termination is observed in the late reflections to occur at about 0.4 seconds. This corresponds to a round trip distance of around 140 meters, roughly the distance from the test section to the downstream wall of turn 3. A more gradual roll-off is observed at $2520 \mathrm{~Hz}$, suggesting multiple reflection paths. At $5 \mathrm{kHz}$ the late reflections are more about $18 \mathrm{~dB}$ down and at $12.7 \mathrm{kHz}$ the late reflections were not observed down to the microphone system noise floor more than $25 \mathrm{~dB}$ below the initial noise level.

Based on the suggestion by Spalt et al., ${ }^{21}$ who recently conducted an acoustic calibration in the NASA Langley 14- by 22-Foot Subsonic Tunnel, the interrupted noise results were used to calculate the Effective Signal to Noise Ratio (ESNR), as shown in Figure 12 (b). This is the measured level difference in decibels between the starting noise level and the start of roll-off due to reflections. This should be the difference in sound level between the incident sound and the reflected sound. The difference between the starting noise level and the background noise level is denoted the Reverberation Time Signal to Noise Ratio (RTSNR) for convenience. Both are plotted for one-third octave band frequencies in Figure 13. When the ESNR is not measurably less than the RTSNR, the ESNR cannot be determined accurately. This happens both at low frequencies, where the sound source produces only a very weak signal, and at high frequencies, where the reflections are too weak to measure. If the ESRN and RTSNR are essentially the same, the RTSNR can be considered to set a lower bound for the ESNR. Figure 13 shows that reflections in the 9x15 LSWT test 


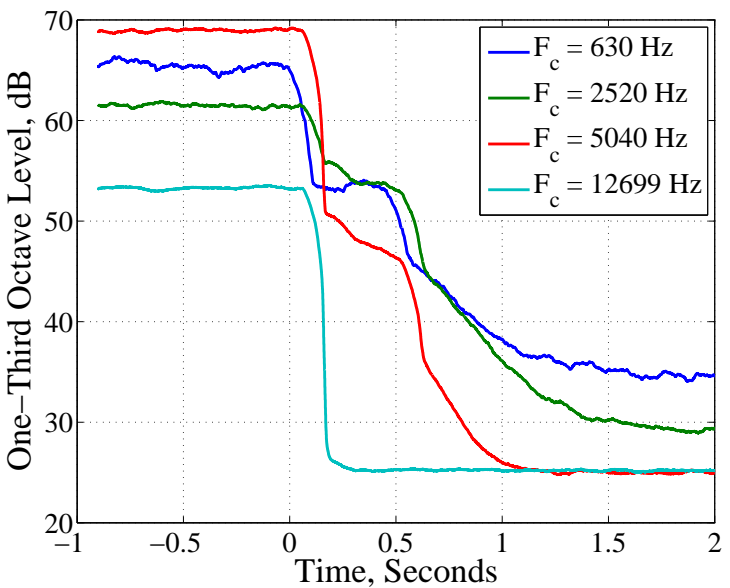

(a)

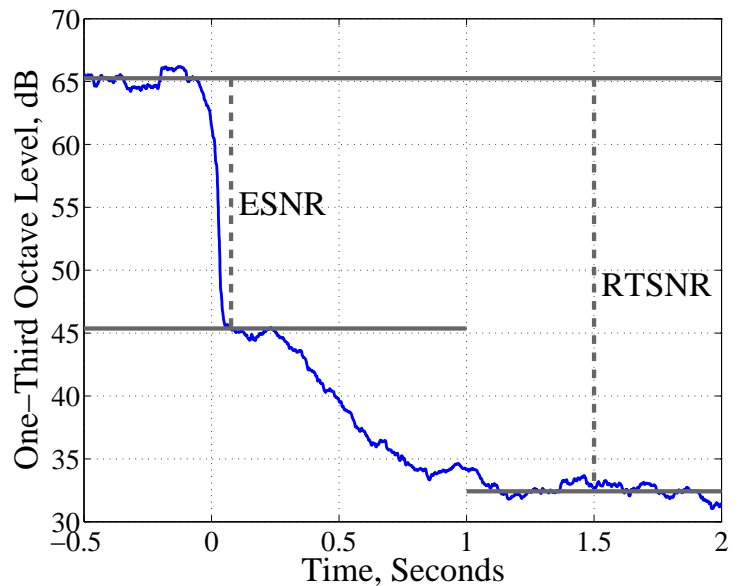

(b)

Figure 12. (a) Example interrupted noise measurements. (b) Calculating Effective Signal to Noise Ratio.

section can be expected to be at least $15 \mathrm{~dB}$ below the incident signal between $200 \mathrm{~Hz}$ and $20 \mathrm{kHz}$, which is consistant with the findings of Dahl and Woodward who found absorption greater than 0.95 from $250 \mathrm{~Hz}$ to $4 \mathrm{kHz} .{ }^{6}$ Reflections of $5 \%$ would be 13 decibels below the incident signal. This method seems to provide a simple and straightford global metric for the anechoic quality of the test section walls.

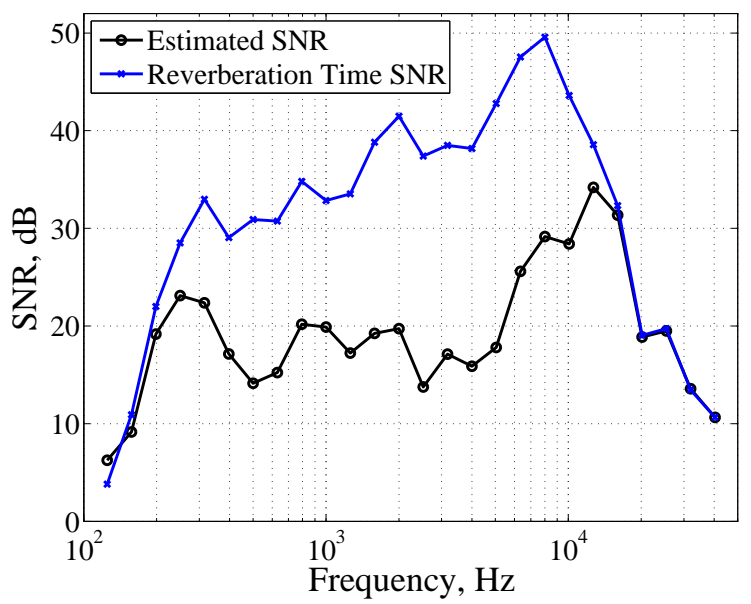

Figure 13. Estimated Signal to Noise Ratio.

\section{Summary}

A significant effort has recently been invested in quantifying the noise environment in the 9x15 LSWT. Noise measurements were taken throughout the tunnel loop and with the tunnel at different operating conditions. A significant contribution to the background noise level measured in the test section is now believed to be due to airflow over the perforated plate that forms the acoustic treatment. This led to an investigation of sample panels in a specialized roughness noise facility. The anechoic quality of the facility was also investigated, using an interrupted noise method. The Effective Signal to Noise Ratio seems to be a simple global metric that could be easily measured again in the future to ensure the acoustic treatment is still performing as expected. 


\section{Acknowledgments}

The NASA Environmentally Responsible Aviation Project funded the 9x15 LSWT background noise measurements. The NASA Aeronautics Test Program funded the roughness noise testing at Virginia Tech. The writing of this report was funded by the NASA Advanced Air Transportation Technologies Project.

\section{References}

${ }^{1}$ Beranek, L. L., Labate, S., and Ingard, U., "Acoustical Treatment of the NACA 8- by 6-Foot Supersonic Propulsion Wind Tunnel," NACA TN-3378, June 1955.

${ }^{2}$ Yuska, J. A., james H. Diedrich, and Clough, N., "Lewis 9- by 15-Foot V/STOL Wind Tunnel," NASA/TM-X-2305, July 1971.

${ }^{3}$ Soeder, R. H., "NASA Lewis 9- by 15-foot Low-Speed Wind Tunnel User Manual," NASA TM-106247, 1993.

${ }^{4}$ Rentz, P. E., "Softwall acoustical characteristics and measurement capabilities of the NASA Lewis 9x15 foot low speed wind tunnel," NASA CR-135026, 1976.

${ }^{5}$ Arrington, A. E. and Gonsalez, J. C., "Calibration of the NASA Lewis Research Center 9- by 15-Foot Low Speed Wind Tunnel (1994 Test)," NASA CR-195438, 1997.

${ }^{6}$ Dahl, M. D. and Woodward, R. P., "Comparison between design and installed acoustic characteristics of NASA Lewis 9by 15-foot low-speed wind tunnel acoustic treatment," NASA TP-2966, 1990.

${ }^{7}$ Dahl, M. D. and Woodward, R. P., "Acoustical Evaluation of the NASA Lewis 9- by 15-Foot Low Speed Wind Tunnel," NASA TP-3274, 1992

${ }^{8}$ Woodward, R. P., Dittmar, J. H., Hall, D. G., and Kee-Bowling, B., "Background Noise Levels Measured in the NASA Glenn 9- by 15-Foot Low-Speed Wind Tunnel," AIAA-95-0720 / NASA TM-106817, 1995.

${ }^{9}$ Woodward, R. P., Loeffler, I. J., and Dittmar, J. H., "Measured Far-Field Flight Noise of a Counterrotation Turboprop at Cruise Conditions," NASA/TM-101383, January 1989.

${ }^{10}$ Stephens, D. B. and Envia, E., "Acoustic Shielding for a Model Scale Counter-rotation Open Rotor. AIAA-2011-2940," 17th AIAA/CEAS Aeroacoustics Conference,, Portland, Oregon, USA, 5-8 June 2011.

${ }^{11}$ Woodward, R. P., Hughes, C. E., Jeracki, R. J., and Miller, C. J., "Fan Noise Source Diagnostic Test - Far-field Acoustic Results," AIAA 2002-2427, 2002.

${ }^{12}$ Balan, C. and Hoff, G. E., "Propulsion Simulator for High Bypass Turbofan Performance Evaluation," 1993 SAE Aerospace Atlantic Conference \& Exposition, Dayton Ohio, April 20-23, 1993.

${ }^{13}$ Duell, E., Yen, J., Walter, J., and Arnette, S., "Boundary Layer Noise in Aeroacoustic Wind Tunnels, AIAA 2004-1028," 42nd AIAA Aerospace Sciences Meeting and Exhibit, Reno, Nevada, USA, 5-8 January 2004.

${ }^{14}$ Hersh, A. S., "Experimental Investigation of Surface Roughness Generated Flow Noise AIAA 87-0786," 8th AIAA Aeroacoustics Conference, Atlanta, Georgia, 11-13 April, 1983.

${ }^{15}$ Becks, E. A., "Enhancing the Capabilities of NASA Lewis' $8 \times 6 / 9 \times 15$ Wind Tunnel Complex Through Drive System Modifications," NASA TM-106840, January 1995.

${ }^{16}$ Becks, E. A., Bencic, T. J., and Blumenthal, P. Z., "Improved Speed Control System for an 87,000 HP Wind Tunnel Drive," NASA TM-106840, January 1995.

${ }^{17}$ Alexander, W. N., Sound from Rough Wall Boundary Layers, Ph.D. thesis, Aerospace and Ocean Engineering Dept., Virginia Tech, Blacksburg, VA, 2011, 2011, http://scholar.lib.vt.edu/theses/available/etd-10112011-085924.

${ }^{18}$ Alexander, W. N. and Devenport, W., "Noise from Boundary Layer Flow over Fabric Covered Perforate Panels AIAA 2014-2908," 20th AIAA/CEAS Aeroacoustics Conference, Atlanta, Georgia, 16-20 June 2014.

${ }^{19}$ Liu, Y. and Dowling, A. P., "Assessment of the Contribution of Surface Roughness to Airframe Noise," AIAA Journal, Vol. 45, No. 4, April 2007, pp. 855-869.

${ }^{20}$ International Organization for Standardization, "Acoustics Measurement of sound absorption in a reverberation room," ISO 354, 2003.

${ }^{21}$ Spalt, T. B., Brooks, T. F., Bahr, C. J., Plassman, G. E., Becker, L. E., and Stead, D. J., "Calibrations of the NASA Langley 14- by 22-Foot Subsonic Tunnel in Acoustic Configuration AIAA 2014-2344," 20th AIAA/CEAS Aeroacoustics Conference, Atlanta, Georgia, 16-20 June 2014. 